TRANSACTIONS OF THE

AMERICAN MATHEMATICAL SOCIETY

Volume 358, Number 1, Pages 251-265

S 0002-9947(05)03697-4

Article electronically published on January 21, 2005

\title{
SOME COUNTEREXAMPLES TO A GENERALIZED SAARI'S CONJECTURE
}

\author{
GARETH E. ROBERTS
}

\begin{abstract}
For the Newtonian $n$-body problem, Saari's conjecture states that the only solutions with a constant moment of inertia are relative equilibria, solutions rigidly rotating about their center of mass. We consider the same conjecture applied to Hamiltonian systems with power-law potential functions. A family of counterexamples is given in the five-body problem (including the Newtonian case) where one of the masses is taken to be negative. The conjecture is also shown to be false in the case of the inverse square potential and two kinds of counterexamples are presented. One type includes solutions with collisions, derived analytically, while the other consists of periodic solutions shown to exist using standard variational methods.
\end{abstract}

\section{INTRODUCTION}

We investigate a generalized version of Saari's conjecture for an $n$-body problem with power-law potential functions. Let $\mathbf{q}=\left(\mathbf{q}_{1}, \mathbf{q}_{2}, \ldots, \mathbf{q}_{n}\right) \in \mathbb{R}^{3 n}$ represent the coordinates of the $n$ bodies in position space with respective masses $m_{i}$. The family of potential functions $U(\mathbf{q})$ of interest for this work is

$$
U(\mathbf{q})=\sum_{i<j} \frac{m_{i} m_{j}}{r_{i j}^{\alpha}}
$$

where $r_{i j}=\left\|\mathbf{q}_{i}-\mathbf{q}_{j}\right\|$ measures the distance between the $i$-th and $j$-th bodies, and $\alpha>0$ is a real parameter. We will usually treat the masses $m_{i}$ as positive, real numbers. However, interesting solutions arise when at least one of the masses is taken to be negative. Let $M=m_{1}+\cdots+m_{n}$ represent the total mass. Without loss of generality we assume that the center of mass

$$
\overline{\mathbf{q}}=\frac{1}{M} \sum_{i=1}^{n} m_{i} \mathbf{q}_{i}
$$

is always at the origin.

The case $\alpha=1$ corresponds to the classical $n$-body problem of celestial mechanics. Note that $U(\mathbf{q})$ is a homogeneous potential of degree $-\alpha$. The equations of motion for this potential function are

$$
m_{i} \ddot{\mathbf{q}}_{i}=\frac{\partial U}{\partial \mathbf{q}_{i}}=-\alpha \sum_{i \neq j} \frac{m_{i} m_{j}\left(\mathbf{q}_{i}-\mathbf{q}_{j}\right)}{r_{i j}^{\alpha+2}} .
$$

Received by the editors September 12, 2003 and, in revised form, February 9, 2004.

2000 Mathematics Subject Classification. Primary 70F10, 70F15; Secondary 37J45.

Key words and phrases. Saari's conjecture, $n$-body problems, relative equilibria, Hamiltonian systems.

(C)2005 American Mathematical Society Reverts to public domain 28 years from publication 
The key quantity for this work is the moment of inertia $I$, measuring the size of the system with respect to the center of mass, given by

$$
I(\mathbf{q})=\frac{1}{2} \sum_{i=1}^{n} m_{i}\left\|\mathbf{q}_{i}\right\|^{2} .
$$

The system of differential equations determined by (1.2) is a Hamiltonian system with Hamiltonian function

$$
H=\frac{1}{2} \sum_{i=1}^{n} m_{i}\left\|\dot{\mathbf{q}}_{i}\right\|^{2}-U(\mathbf{q}) .
$$

One simple but very important type of solution to these physical systems is a relative equilibrium, named in part because it is a fixed solution in a rotating coordinate system. To define these special trajectories, we first introduce the notion of a central configuration, which plays an important role in the counterexample developed in Section 2 .

Definition 1.1. A central configuration for a system of type (1.2) is a configuration $\mathbf{x} \in \mathbb{R}^{3 n}$ which satisfies the algebraic equations

$$
\nabla U(\mathbf{x})+\lambda \nabla I(\mathbf{x})=0
$$

for some value of $\lambda$.

From the definition above, a central configuration is a critical point of the potential function $U$ restricted to the "mass ellipsoid" defined as the level set $I=k$, where $\lambda$ plays the role of a Lagrange multiplier. Classifying central configurations and illuminating their properties is an on-going subject of interest in celestial mechanics [8, 15. Central configurations can be collinear, coplanar or spatial.

Suppose for the moment that we are in the planar $n$-body problem, with the position vector $\mathbf{q} \in \mathbb{R}^{2 n}$. Denote $R(t)$ as the rotation matrix

$$
R(t)=\left[\begin{array}{rr}
\cos t & -\sin t \\
\sin t & \cos t
\end{array}\right]
$$

and let $R(t) \mathbf{q}=\left(R(t) \mathbf{q}_{1}, R(t) \mathbf{q}_{2}, \ldots, R(t) \mathbf{q}_{n}\right)$. Any planar central configuration $\mathbf{x}$ begets a family of homographic solutions that preserve the shape of the configuration. Those solutions for which the size is also constant are called relative equilibria.

Definition 1.2. A relative equilibrium for a planar Hamiltonian system of type (1.2) is a solution of the form $\mathbf{q}(t)=R(\omega t) \mathbf{x}$.

It follows by substituting $\mathbf{q}(t)=R(\omega t) \mathbf{x}$ into (1.2) that $\mathbf{x}$ must be a planar central configuration with multiplier $\lambda=\omega^{2}$. A relative equilibrium is a periodic solution whose initial configuration (determined by $\mathbf{x}$ ) is rigidly rotated about the center of mass (in this case the origin). While spatial central configurations exist, it is not possible with positive masses to have a purely spatial (non-coplanar) relative equilibrium.

Note that the equations for a planar central configuration are invariant under rotation. Specifically, if $R$ is a $2 \times 2$ orthogonal matrix and $\mathbf{x}$ is a central configuration with multiplier $\lambda$, then $R \mathbf{x}$ is also a central configuration with the same 
multiplier. When counting solutions to (1.3) for a fixed multiplier $\lambda$, it is standard to identify solutions which are identical under rotation and refer to equivalence classes of central configurations. Holding $\lambda$ fixed enforces a particular size on the configuration, so we can ignore scalar changes in the positions of the bodies. For our purposes, a relative equilibrium will always denote a particular solution to the differential equations defined by (1.2), while an equivalence class of relative equilibria refers to the set of all planar central configurations that are equivalent under rotation.

In 1970, Don Saari made a wonderful conjecture regarding trajectories of a fixed size: Every solution of the Newtonian n-body problem that has a constant moment of inertia is a relative equilibrium [14. No proof or counterexample for the planar case $n \geq 4$ or for the spatial case $n \geq 3$ has been offered. If Saari's conjecture were true, it would be a remarkable fact. Imposing a restriction on the size of a solution, seemingly a minor constraint in a many-body problem, forces that solution to become a rigid rotation.

The conjecture has recently generated a good deal of scholarly interest, in part due to a talk given by Chris McCord at the Midwest Dynamical Systems Conference held at the University of Cincinnati, October 4-7, 2002. At the meeting McCord announced a computer-assisted proof of the conjecture for the equal mass planar 3-body problem 6]. Rick Moeckel has developed an interesting computer-assisted proof using BKK theory and algebraic geometry for the planar three-body problem using mutual distances as coordinates [7. Moeckel's work applies to any choice of masses (including negative values) as long as $m_{i} \neq 0 \forall i$ and $M \neq 0$. Jerry Marsden proposed a generalized version of Saari's conjecture at the same Midwest meeting: For a simple mechanical system with symmetry on the configuration manifold, the locked inertia tensor $I(\mathbf{q})$ is constant along a solution if and only if $\mathbf{q}$ is a relative equilibrium. Marsden's generalization appears to be more restrictive on the motion than Saari's original conjecture and is not discussed here.

We present several counterexamples to a simple generalization of Saari's conjecture to mechanical systems of type (1.2) with potential function (1.1). We begin by showing that Saari's conjecture fails in the five-body problem for the masses $\left(1,1,1,1,-1 / 2^{\alpha+1}\right)$. The counterexample presented is valid for all $\alpha$ and is based on a continuum of planar central configurations established in 13 . The relationship between Saari's conjecture and a famous question on the finiteness of equivalence classes of relative equilibria is discussed as well. The existence of a counterexample to Saari's conjecture given a continuum of planar central configurations was independently pointed out by Alain Chenciner in the proceedings of the Celestial Mechanics Conference in honor of Don Saari [2].

In Section 3 we show that Saari's conjecture is false for the inverse square potential $(\alpha=2)$. This potential has been well studied, dating back to the works of Newton and Jacobi [5]. One counterexample is derived in Section 3.1 for the equal mass 4-body problem. The configuration for this solution is always a rhombus with constant side length, beginning and ending in binary collision. In Section 3.2 we use standard variational techniques to prove the existence of periodic solutions with constant inertia which are not relative equilibria. Although these examples may cast a shadow of doubt on the validity of Saari's conjecture for the Newtonian $n$-body problem, we are unable to make any definitive claims as to the truthfulness of that conjecture. 


\section{A COntinuUm of Relative EQUilibria}

There is a clear connection between Saari's conjecture and an old question in celestial mechanics posed by Wintner [17] and later re-emphasized by Smale as one of his open problems for the 21st century [16]. The question is whether the number of equivalence classes of relative equilibria in the $n$-body problem is finite or not. To date, finiteness has only been proven for the case $n=3$, for four equal masses [1] and generically when $n=4$ 9. Since planar central configurations can be formulated as critical points of the potential function restricted to the mass ellipsoid $I=k$, an entire curve of such critical points would present a tempting counterexample to Saari's conjecture. In a relative equilibrium solution, each configuration along the orbit is also a critical point of $U$ restricted to $I=k$.

Although the two conjectures are certainly not equivalent, there is a relationship worth pursuing. It is straightforward to see that a continuum of relative equilibria will provide a solution to the $n$-body problem if and only if each component is the solution to the same harmonic oscillator equation.

Lemma 2.1. Suppose that $\mathbf{q}(s)$ is a one-parameter family of planar central configurations, that is, $\mathbf{q}(s)$ satisfies (1.3) for each value of $s$, for the same multiplier $\lambda>0$, with $I(\mathbf{q}(s))=k$ a constant. Then, $\mathbf{q}(s)$ is a solution to the generalized $n$-body problem described by system (1.2) if and only if each component of $\mathbf{q}_{i}(s)$ can be expressed as $\xi_{1} \cos (\sqrt{\lambda} s)+\xi_{2} \sin (\sqrt{\lambda} s)$.

Proof. This follows directly from (1.3). Substitution of $\mathbf{q}(s)$ into the differential equation (1.2) yields

$$
m_{i} \frac{d^{2} \mathbf{q}_{i}}{d s^{2}}=\frac{\partial U(\mathbf{q}(s))}{\partial \mathbf{q}_{i}}=-\lambda \frac{\partial I(\mathbf{q}(s))}{\partial \mathbf{q}_{i}}=-\lambda m_{i} \mathbf{q}_{i} .
$$

Thus, in order for $\mathbf{q}(s)$ to be a solution, each of its $2 n$ components must satisfy the standard harmonic oscillator equation with frequency $\sqrt{\lambda} /(2 \pi)$. This is both a necessary and sufficient condition. Note that any relative equilibrium solution satisfies this requirement since each component is the product of the orthogonal matrix $R(\sqrt{\lambda} s)$ and a constant vector.

Under the same hypotheses as the lemma, the power-law potential function $U$ is constant along $\mathbf{q}(s)$ taking the value $2 \lambda k / \alpha$. This is easily seen by taking the dot product of $\mathbf{q}(s)$ with (1.3) and using the fact that $U$ is homogeneous of degree $-\alpha$ and $I$ is homogeneous of degree 2 . We then obtain

$$
-\alpha U(\mathbf{q}(s))+2 \lambda I(\mathbf{q}(s))=0
$$

or $U(\mathbf{q}(s))=2 \lambda k / \alpha$.

While it seems unlikely that a continuum of relative equilibria equivalence classes exists in the classical $n$-body problem, there is an example if the coefficients $m_{i}$ are allowed to take on different signs. In 13 we presented such an example for the Newtonian 5-body problem. For the case of the generalized potential function $U$ in (1.1), this continuum of central configurations occurs in the planar 5-body problem with masses $m_{1}=m_{2}=m_{3}=m_{4}=1$ and $m_{5}=-1 / 2^{\alpha+1}$.

To begin, we describe a symmetric configuration by setting $\mathbf{q}_{2}=-\mathbf{q}_{1}, \mathbf{q}_{4}=$ $-\mathbf{q}_{3}$ and $\mathbf{q}_{5}=(0,0)$. By the perpendicular bisector theorem (see [8]), for the configuration to be central and non-collinear, it follows that the positions of the 3rd and 4 th bodies must lie on the perpendicular bisector of the segment joining $\mathbf{q}_{1}$ 


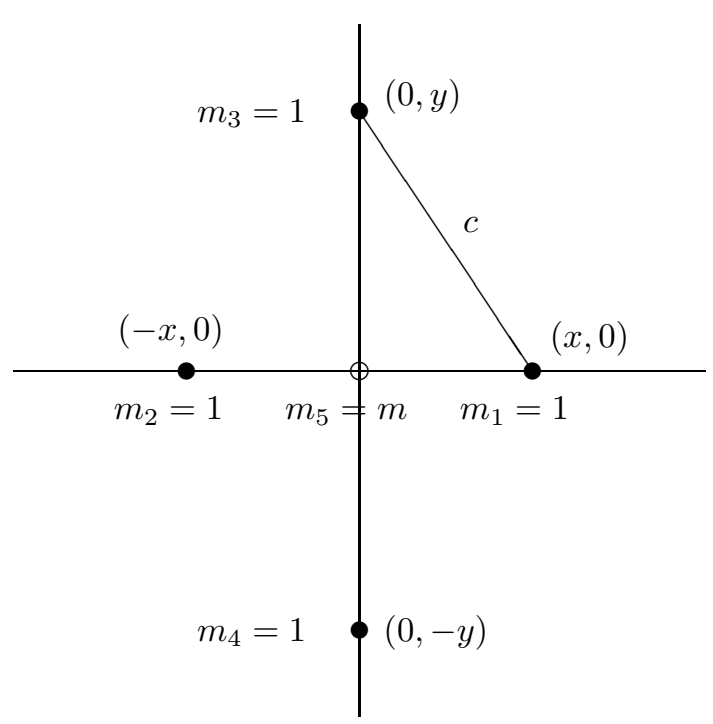

Figure 1 . The $1+$ rhombus family of relative equilibria, where $\theta=0$ and $m=-1 / 2^{\alpha+1}$.

and $\mathbf{q}_{2}$. This places the first four bodies at the vertices of a rhombus. Identifying $\mathbb{R}^{2}$ with $\mathbb{C}$, we set $\mathbf{q}_{1}=x e^{i \theta}$ and $\mathbf{q}_{3}=y i e^{i \theta}$, with $x>0, y>0$ and $\theta$ as real variables (see Figure 1 where $\theta=0$ ). It turns out that for the values of the masses stated above, this yields a planar central configuration for all $\alpha$ and any choice of $x, y$ and $\theta$.

Theorem 2.2. Let $m_{1}=m_{2}=m_{3}=m_{4}=1$ and $m_{5}=-1 / 2^{\alpha+1}$. Set $\mathbf{q}_{1}=$ $x e^{i \theta}, \mathbf{q}_{2}=-\mathbf{q}_{1}, \mathbf{q}_{3}=y i e^{i \theta}, \mathbf{q}_{4}=-\mathbf{q}_{3}$ and $\mathbf{q}_{5}=0$. This configuration is central for all $\alpha$ with multiplier $\lambda=2 \alpha / c^{\alpha+2}$ where $c=\sqrt{x^{2}+y^{2}}$.

Proof. It is a straightforward calculation to check that this setup satisfies equation (1.3) without any restrictions on $x, y$ or $\theta$. The mutual distances are $r_{12}=$ $2 x, r_{34}=2 y, r_{13}=r_{14}=r_{23}=r_{24}=c, r_{15}=r_{25}=x$ and $r_{35}=r_{45}=y$. The symmetry implies that the forces on the first and second bodies differ only in sign, as do the forces on the third and fourth bodies. The forces on the central body cancel out to zero. Equation (1.3) then reduces to the two equations

$$
\begin{aligned}
& -\alpha\left(\frac{2 x e^{i \theta}}{(2 x)^{\alpha+2}}+\frac{x e^{i \theta}-y i e^{i \theta}}{c^{\alpha+2}}+\frac{x e^{i \theta}+y i e^{i \theta}}{c^{\alpha+2}}-\frac{x e^{i \theta}}{2^{\alpha+1} x^{\alpha+2}}\right)+\lambda x e^{i \theta}=0, \\
& -\alpha\left(\frac{2 y i e^{i \theta}}{(2 y)^{\alpha+2}}+\frac{y i e^{i \theta}-x e^{i \theta}}{c^{\alpha+2}}+\frac{y i e^{i \theta}+x e^{i \theta}}{c^{\alpha+2}}-\frac{y i e^{i \theta}}{2^{\alpha+1} y^{\alpha+2}}\right)+\lambda y i e^{i \theta}=0 .
\end{aligned}
$$

Dividing the first equation by $x e^{i \theta}$ and the second by $y i e^{i \theta}$ yields

$$
\begin{aligned}
& -\alpha\left(\frac{2}{(2 x)^{\alpha+2}}+\frac{2}{c^{\alpha+2}}-\frac{1}{2^{\alpha+1} x^{\alpha+2}}\right)+\lambda=0, \\
& -\alpha\left(\frac{2}{(2 y)^{\alpha+2}}+\frac{2}{c^{\alpha+2}}-\frac{1}{2^{\alpha+1} y^{\alpha+2}}\right)+\lambda=0 .
\end{aligned}
$$

Remarkably, both of these last equations are satisfied with the correct choice of the multiplier $\lambda=2 \alpha / c^{\alpha+2}$, independent of the values of $x, y$ or $\theta$. 
Note that the inertia for any configuration in the continuum is given by $I=$ $x^{2}+y^{2}=c^{2}$. Holding the inertia constant will require the side of the rhombus to remain constant as well. The potential function becomes a function of two variables and $U(x, y)=4 / c^{\alpha}$ is constant for any choice of $x, y$ satisfying $x^{2}+y^{2}=c^{2}$. As a check, (2.1) then gives the value of $\lambda$ listed in the theorem.

Physically, the reason the continuum works is that the negative central mass acts as a repeller canceling out the forces along the diagonals of the rhombus. The corresponding centrifugal force needed to maintain the equilibrium works out to be the same for the first and second bodies as it is for the third and fourth, essentially because the equations for each pair of bodies decouples. It should be pointed out that $I(\mathbf{q})$ is no longer positive definite when negative masses are allowed. This means that requiring solutions to satisfy $I=c^{2}$ constrains them to a mass hyperboloid rather than a mass ellipsoid. Thus the potential function can remain constant even if the configuration is arbitrarily close to triple collision.

To find a solution to the generalized $n$-body problem with constant inertia $I=c^{2}$, we must choose a path $\mathbf{q}(s)$ through the continuum such that each component satisfies the same harmonic oscillator equation

$$
\frac{d^{2} \mathbf{q}_{i}}{d s^{2}}=-\lambda \mathbf{q}_{i}
$$

with $\lambda=2 \alpha / c^{\alpha+2}$. We now calculate all such possible paths for fixed $c$ and $\alpha$.

Substituting $\mathbf{q}_{1}=x e^{i \theta}$ into (2.2) leads to the system of differential equations

$$
\begin{aligned}
\ddot{x}-x \dot{\theta}^{2} & =-\lambda x, \\
2 \dot{x} \dot{\theta}+x \ddot{\theta} & =0 .
\end{aligned}
$$

Multiplying the second equation by $x$ and integrating once leads to the identity $x^{2} \dot{\theta}=\omega_{1}$, where $\omega_{1}$ is an integration constant. Similar equations are obtained for $\mathbf{q}_{3}=y i e^{i \theta}$ resulting in the system

$$
\begin{aligned}
\ddot{x} & =\frac{\omega_{1}^{2}}{x^{3}}-\lambda x, \\
\ddot{y} & =\frac{\omega_{2}^{2}}{y^{3}}-\lambda y, \\
\dot{\theta} & =\frac{\omega_{1}}{x^{2}}=\frac{\omega_{2}}{y^{2}}
\end{aligned}
$$

with two integration constants $\omega_{1}$ and $\omega_{2}$. This system must be solved subject to the constraint $x^{2}+y^{2}=c^{2}$. Multiplying this constraint by $\dot{\theta}$ and using (2.5), we see that $\dot{\theta}=\left(\omega_{1}+\omega_{2}\right) / c^{2}$. There are two cases depending on whether the angle $\theta$ moves uniformly or is constant.

Case 1: $\omega_{1} \omega_{2} \neq 0$. Equation (2.5) implies that $\omega_{1}$ and $\omega_{2}$ have the same sign, that is, $\omega_{1} \omega_{2}>0$. This in turn implies that $\omega_{1}+\omega_{2} \neq 0$ and that $\omega_{1} /\left(\omega_{1}+\omega_{2}\right)$, $\omega_{2} /\left(\omega_{1}+\omega_{2}\right)$ are positive constants less than one. Equation (2.5) then yields

$$
x=\sqrt{\omega_{1} /\left(\omega_{1}+\omega_{2}\right)} c \text { and } y=\sqrt{\omega_{2} /\left(\omega_{1}+\omega_{2}\right)} c
$$

both constant, so that the motion is a relative equilibrium. Substituting these values for $x$ and $y$ into the differential equations (2.3) and (2.4) gives the condition $\omega_{1}+\omega_{2}=\operatorname{sgn}\left(\omega_{1}\right) \sqrt{\lambda} c^{2}$. Therefore, the angular velocity of the relative equilibrium depends only on $c$ (and $\alpha$ ) and a choice of sign, $\theta(s)= \pm \sqrt{\lambda} s+\theta_{0}$. There are 
essentially two free parameters, $\omega_{1}$, controlling the breadth of the rhombus and $\theta_{0}$, specifying a starting angle.

Case 2: $\omega_{1} \omega_{2}=0$. Note that since $x$ and $y$ are both positive, (2.5) shows that

$$
\omega_{1}=0 \quad \text { iff } \quad \omega_{2}=0 \text { iff } \dot{\theta}=0 .
$$

In this case we obtain the simple system

$$
\ddot{x}=-\lambda x, \quad \ddot{y}=-\lambda y .
$$

Differentiating the constraint $x^{2}+y^{2}=c^{2}$ once yields the relation

$$
x \dot{x}=-y \dot{y} .
$$

Another differentiation and substitution using (2.6) gives $\dot{x}^{2}+\dot{y}^{2}=\lambda c^{2}$. Finally, replacing $\dot{y}$ in this last equation with the value given in (2.7) yields

$$
y=\frac{1}{\sqrt{\lambda}}|\dot{x}| \text {. }
$$

Of course we know that $x$ is a simple harmonic oscillator

$$
x(s)=k_{1} \cos \left(\sqrt{\lambda} s-k_{2}\right)
$$

for some constants $k_{1}$ and $k_{2}$. Substitution into (2.8) gives $y(s)=\left|k_{1} \sin \left(\sqrt{\lambda} s-k_{2}\right)\right|$ and since $x$ and $y$ are constrained to a circle of radius $c$, we must have $\left|k_{1}\right|=c$. Varying $k_{2}$ results in a reparameterization of time, thus we set $k_{2}=0$. There are two types of motion depending on which pair of bodies comes from or heads toward triple collision. The side length of the rhombus remains constant during the motion but one of the diagonals collapses as the other is enlarged.

The motion for solution Type A is described by

$$
x(s)=c \cos (\sqrt{\lambda} s), \quad y(s)=c \sin (\sqrt{\lambda} s), \quad 0<s<\frac{\pi}{2 \sqrt{\lambda}},
$$

which has bodies 3 and 4 coming from a triple collision at the origin and bodies 1 and 2 heading towards a triple collision. The motion for solution Type B is described by

$$
x(s)=c \cos (\sqrt{\lambda} s), \quad y(s)=-c \sin (\sqrt{\lambda} s), \quad-\frac{\pi}{2 \sqrt{\lambda}}<s<0,
$$

which has bodies 1 and 2 coming from a triple collision at the origin and bodies 3 and 4 heading towards a triple collision. The value of $\theta(s) \equiv \theta_{0}$ is arbitrary and simply controls the angle of the diagonal between bodies 1 and 2 . The two motions are identical under a 90 -degree rotation of $\theta_{0}$ and a relabeling of the masses. Clearly, neither type of motion corresponds to a relative equilibrium.

Theorem 2.3. For each $c, \alpha>0$, there exist two types of solutions to the generalized 5 -body problem with masses $\left(1,1,1,1,-1 / 2^{\alpha+1}\right)$ which have a constant moment of inertia $I=c^{2}$ but are not relative equilibria. At any fixed time the configuration of bodies forms a planar central configuration. Each motion begins and ends with triple collision.

Remark 2.4. The calculations above show that if the moment of inertia is held constant, then solutions of Types A and B are the only possible non-relative equilibrium motions passing through the continuum of central configurations. Other interesting motions are possible if the inertia is allowed to vary, but these will not be counterexamples to Saari's conjecture. 
Corollary 2.5. If negative masses are permitted, Saari's conjecture fails in the generalized 5-body problem.

One important consequence of the theorem and its corollary is that any algebraic proof of Saari's conjecture for the Newtonian five-body problem will need to restrict the coefficients $m_{i}$ to be positive or else yield conditions on the masses which necessarily exclude the case above. Straightforward applications of techniques from algebraic geometry may not succeed without being aware of this restriction.

\section{The inVerse square Potential}

In this section we explain why Saari's conjecture fails in the case of the inverse square potential $U(\mathbf{q})=\sum m_{i} m_{j} / r_{i j}{ }^{2}$. There is a relatively large set (codimension 2) of choices for the initial positions and velocities of the $n$ bodies leading to solutions with a constant moment of inertia which are not relative equilibria. One specific example is presented in Section 3.1 for the case of four equal masses. It is a rhombus with constant side length, beginning and ending in a binary collision. The strong amount of symmetry present allows us to explicitly calculate a simple expression for this solution. The existence of families of periodic solutions with constant inertia is easily proven using variational methods.

Due to the well-known Lagrange-Jacobi identity [5], it is clear that for the special case $\alpha=2$, solutions with constant inertia are readily found. To derive it, treat the inertia $I$ as a function of $t$ and differentiate once obtaining

$$
\dot{I}=\sum_{i=1}^{n} m_{i} \mathbf{q}_{i} \cdot \dot{\mathbf{q}}_{i}
$$

Using the fact that $U$ is homogeneous of degree $-\alpha$, a further differentiation yields the Lagrange-Jacobi identity

$$
\ddot{I}=\sum_{i=1}^{n} m_{i}\left\|\dot{\mathbf{q}}_{i}\right\|^{2}+\sum_{i=1}^{n} \mathbf{q}_{i} \cdot \frac{\partial U}{\partial \mathbf{q}_{i}}=2 H+(2-\alpha) U .
$$

Therefore, if $\alpha \neq 2$, the moment of inertia $I$ being constant along a solution forces the potential function $U$ to be constant as well. This added constraint on the motion is used by McCord and Moeckel in their proofs for the Newtonian potential function. For the case $\alpha=2$, it is possible to have solutions containing collisions (infinite potential) while still having constant moment of inertia.

Setting $\alpha=2$, two integrations of (3.1) yields the important formula

$$
I(t)=H t^{2}+c_{1} t+c_{2},
$$

where $c_{1}$ and $c_{2}$ are integration constants. This equation appears in Jacobi's famous "Vorlesungen über dynamik" [5]. If $\mathbf{q}(t)$ is a periodic solution, $I(t)$ must also be periodic, from which it follows that $H=c_{1}=0$ and $I(t)$ is necessarily constant. Therefore, any periodic solution for the inverse square potential mechanical system necessarily has constant moment of inertia [3]. Any such solution which is not a relative equilibrium is a counterexample to the generalized Saari's conjecture.

Note that $c_{1}=\dot{I}(0)$. If the initial positions and velocities are chosen so that $H=$ $c_{1}=0$, then the corresponding motion will have constant inertia. The constraints $H=0$ and $\dot{I}=0$ generically define a codimension two manifold in the phase space of the $n$-body problem $\left(\mathbb{R}^{3 n}-\Sigma\right) \times \mathbb{R}^{3 n}$, where $\Sigma$ is the set of collisions 
$\Sigma=\left\{\mathbf{q} \in \mathbb{R}^{3 n}: \mathbf{q}_{i}=\mathbf{q}_{j}\right.$ for some $\left.i \neq j\right\}$. It is not difficult to find initial conditions that satisfy these two constraints.

Theorem 3.1. Let $n \geq 3$. Suppose that $n-1$ initial positions and velocities are arbitrarily chosen for $n-1$ bodies, with the minor requirement that

$$
\left(\mathbf{q}_{i}(0)-\mathbf{q}_{j}(0)\right) \cdot\left(\dot{\mathbf{q}}_{i}(0)-\dot{\mathbf{q}}_{j}(0)\right) \neq 0
$$

for some pair of distinct indices $i$ and $j$. Then, it is possible to specify the location and velocity of the $n$-th body so that $H=\dot{I}=0$. The solution satisfying these initial conditions has constant moment of inertia but is not a relative equilibrium.

Proof. Note that in the case $n=2$, the only periodic solutions possible are relative equilibria. This is easily seen for the inverse square Kepler problem since a constant value of $I$ implies a constant length of the position and velocity vectors.

Fix the positions and velocities of the first $n-1$ bodies. The two equations $H=0$ and $\dot{I}=0$ can be written in terms of the unknowns $\mathbf{q}_{n}$ and $\dot{q}_{n}$ as

$$
\begin{aligned}
\beta_{1}+\left\|\mathbf{q}_{n}\right\|\left\|\dot{\mathbf{q}}_{n}\right\| \cos \theta_{n} & =0, \\
\beta_{2}+\left\|\dot{\mathbf{q}}_{n}\right\|^{2}-\tilde{U}\left(\mathbf{q}_{n}\right) & =0,
\end{aligned}
$$

where $\beta_{1}$ and $\beta_{2}$ are real constants depending on $\dot{I}$ and the energy of the $n-1$ fixed bodies, $\theta_{n}$ is the angle between the vectors $\mathbf{q}_{n}$ and $\dot{q}_{n}$, and $\tilde{U}$ is an amended potential function

$$
\tilde{U}\left(\mathbf{q}_{n}\right)=2 \sum_{i=1}^{n-1} \frac{m_{i}}{r_{i n}^{2}} .
$$

Choose the position of the $n$-th body $\mathbf{q}_{n} \neq \mathbf{0}$ such that

$$
\begin{aligned}
\tilde{U}\left(\mathbf{q}_{n}\right)-\beta_{2} & >0, \\
\text { and }\left\|\mathbf{q}_{n}\right\| \sqrt{\tilde{U}\left(\mathbf{q}_{n}\right)-\beta_{2}} & \geq\left|\beta_{1}\right| .
\end{aligned}
$$

If $\beta_{2}$ is large and positive this requires that $\mathbf{q}_{n}$ be chosen to lie within some small neighborhood of one of the first $n-1$ bodies so as to make the amended potential $\tilde{U}$ large enough. The inequalities above in the variable $\mathbf{q}_{n}$ determine some closed region in space, disconnected if $\beta_{2}$ is sufficiently large and positive.

Now set

$$
\left\|\dot{q}_{n}\right\|=\sqrt{\tilde{U}\left(\mathbf{q}_{n}\right)-\beta_{2}} \quad \text { and } \quad \theta_{n}=\cos ^{-1}\left(\frac{-\beta_{1}}{\left\|\mathbf{q}_{n}\right\| \sqrt{\tilde{U}\left(\mathbf{q}_{n}\right)-\beta_{2}}}\right) .
$$

As a result of these choices, $H=\dot{I}=0$, so that the moment of inertia $I$ is constant.

The condition (3.2) is needed to ensure that the ensuing motion is not a relative equilibrium. This condition does not depend on the center of mass, an important feature since apriori, $\overline{\mathbf{q}}$ is unknown until $\mathbf{q}_{n}(0)$ is chosen. Suppose that $\mathbf{q}(\mathrm{t})$ is a relative equilibrium motion in $\mathbb{R}^{3 n}$ with center of mass $\overline{\mathbf{q}}(t)$ not necessarily fixed. Then each component can be described by $\mathbf{q}_{i}(t)=P A(\omega t) P^{T} \mathbf{x}_{i}+\overline{\mathbf{q}}(t)$ for some orthogonal matrix $P$ where

$$
A(t)=\left[\begin{array}{cc}
R(t) & 0 \\
0 & 1
\end{array}\right]
$$

and $R(t)$ is the standard $2 \times 2$ rotation matrix (1.4). Using the fact that $A(t) \mathbf{x}$ is orthogonal to $\frac{d}{d t} A(t) \mathbf{x}$ for any vector $\mathbf{x}$, it is straightforward to compute that 
the vectors $\mathbf{q}_{i}(t)-\mathbf{q}_{j}(t)$ and $\dot{\mathbf{q}}_{i}(t)-\dot{\mathbf{q}}_{j}(t)$ are orthogonal for all pairs $i, j$ at any time $t$.

Remark 3.2. In the spirit of Saari's conjecture, since the moment of inertia $I$ is being measured with respect to the origin, we should also require that the center of mass in any counterexample be fixed at the origin. This is not necessarily the case given a solution $\mathbf{q}(t)$ satisfying $H=\dot{I}=0$. However, since the size of the system measured from the origin is constant, it follows that $\overline{\mathbf{q}}$ is constant. Consider the translated solution $\mathbf{q}(t)-\overline{\mathbf{q}}$ with center of mass at the origin. It is straightforward to show that both $H$ and $\dot{I}$ vanish along this solution as well, due to the invariance of $H$ under translation and the fact that $\dot{\overline{\mathbf{q}}}=\mathbf{0}$. Therefore, any solution with constant $I$ can be translated into another solution with constant $I$ whose center of mass is at the origin.

3.1. An example with collisions. The previous theorem shows that, for any $n \geq 3$ and any choice of masses, there exists an abundance of solutions for the $n$ body system of the inverse square potential with constant $I$ which are not relative equilibria. It proves that the generalized Saari's conjecture fails miserably when $\alpha=2$, but does not provide much insight into the types of motion available as counterexamples.

For the inverse square system, the equations of motion for the $i$-th body are given by

$$
m_{i} \ddot{\mathbf{q}}_{i}=\frac{\partial U}{\partial \mathbf{q}_{i}}=-2 \sum_{i \neq j} \frac{m_{i} m_{j}\left(\mathbf{q}_{i}-\mathbf{q}_{j}\right)}{r_{i j}^{4}} .
$$

We consider the 4-body problem with equal masses $m_{i}=1$ and derive a oneparameter family of solutions to the differential equation (3.3) having constant inertia and containing binary collisions. Our parameter $c>0$ represents the square root of the value of the inertia as well as the side length of the rhombus configuration of the 4 bodies.

Theorem 3.3. Let $c>0$ be any real constant and set $\omega=4 / c^{2}$. The function $\mathbf{q}(t)$ with components $\mathbf{q}_{1}(t)=(x / 2,0), \mathbf{q}_{2}(t)=-\mathbf{q}_{1}(t), \mathbf{q}_{3}(t)=(0, y / 2), \mathbf{q}_{4}(t)=-\mathbf{q}_{3}(t)$ where

$$
x(t)=c(2+\sqrt{5} \sin (\omega t))^{1 / 2}, \quad y(t)=c(2-\sqrt{5} \sin (\omega t))^{1 / 2}
$$

is a solution to the equal mass 4-body problem defined by 3.3 with $m_{i}=1 \forall i$. The moment of inertia for $\mathbf{q}(t)$ is constant with the value $I=c^{2}$.

Proof. One can simply check the solution directly by substituting it back into the differential equation. However, to illustrate the significance of the degree of the potential function, we derive the solution from scratch. Begin by looking for a special rhombus-shaped solution $\mathbf{q}(t)$ to (3.3) of the form $\mathbf{q}_{1}(t)=(x(t) / 2,0), \mathbf{q}_{2}(t)=$ $-\mathbf{q}_{1}(t), \mathbf{q}_{3}(t)=(0, y(t) / 2), \mathbf{q}_{4}(t)=-\mathbf{q}_{3}(t)$. In this setup, with $m_{i}=1 \forall i$, the value of the inertia is

$$
I=\frac{1}{4}\left(x(t)^{2}+y(t)^{2}\right) .
$$

We seek expressions for the unknown functions $x(t)$ and $y(t)$ which satisfy the differential equation while simultaneously being constrained to the circle $x^{2}+y^{2}=$ $4 c^{2}$. The distances between pairs of bodies are $r_{12}=x, r_{34}=y$ and $r_{13}=r_{14}=$ $r_{23}=r_{24}=\sqrt{x^{2}+y^{2}} / 2=c$. 
Note that the parameter $c$ has two meanings. It represents the distance between any two bodies not on the same coordinate axis as well as the square root of the moment of inertia. By fixing the moment of inertia, we force the configuration into a rhombus whose side length remains constant. This decouples the resulting differential equations nicely. Without equal masses, the equations of motion would not decouple. (Holding $I$ constant in general does not force so many mutual distances to be constant.) The advantage of the equal mass case is that the mass dependence in the moment of inertia $I$ becomes trivial and the resulting expression is immediately apparent in the differential equation.

Due to symmetry, the differential equations for the first and second bodies differ only by a sign, as do the equations for the third and fourth bodies. The differential equations for the first and third bodies are

$$
\begin{aligned}
& \left(\begin{array}{c}
\ddot{x} / 2 \\
0
\end{array}\right)=-2\left[\frac{1}{x^{4}}\left(\begin{array}{c}
x \\
0
\end{array}\right)+\frac{1}{c^{4}}\left(\begin{array}{c}
x / 2 \\
-y / 2
\end{array}\right)+\frac{1}{c^{4}}\left(\begin{array}{c}
x / 2 \\
y / 2
\end{array}\right)\right], \\
& \left(\begin{array}{c}
0 \\
\ddot{y} / 2
\end{array}\right)=-2\left[\frac{1}{y^{4}}\left(\begin{array}{c}
0 \\
y
\end{array}\right)+\frac{1}{c^{4}}\left(\begin{array}{c}
-x / 2 \\
y / 2
\end{array}\right)+\frac{1}{c^{4}}\left(\begin{array}{c}
x / 2 \\
y / 2
\end{array}\right)\right] .
\end{aligned}
$$

It is clear that the second component of the equation for the first body is satisfied as is the first component of the third body. We are left with two decoupled secondorder differential equations

$$
\begin{aligned}
& \ddot{x}=-4\left(\frac{1}{x^{3}}+\frac{x}{c^{4}}\right), \\
& \ddot{y}=-4\left(\frac{1}{y^{3}}+\frac{y}{c^{4}}\right) .
\end{aligned}
$$

Multiplying both sides of the above equations by $\dot{x}$ and $\dot{y}$, respectively, and integrating once yields

$$
\begin{aligned}
& \dot{x}^{2}=4\left(\frac{1}{x^{2}}-\frac{x^{2}}{c^{4}}\right)+k_{1}, \\
& \dot{y}^{2}=4\left(\frac{1}{y^{2}}-\frac{y^{2}}{c^{4}}\right)+k_{2},
\end{aligned}
$$

where $k_{1}$ and $k_{2}$ are integration constants to be chosen later.

Next, we change variables by letting $x=\sqrt{u}$ and $y=\sqrt{v}$. This leads to

$$
\begin{aligned}
& \dot{u}^{2}=16\left(1-\frac{u^{2}}{c^{4}}\right)+4 k_{1} u, \\
& \dot{v}^{2}=16\left(1-\frac{v^{2}}{c^{4}}\right)+4 k_{2} v .
\end{aligned}
$$

In our new variables, holding the inertia constant results in the constraint $u+v=$ $4 c^{2}$ which forces $\dot{u}^{2}=\dot{v}^{2}$. Therefore, a necessary condition on the integration constants $k_{1}$ and $k_{2}$ is

$$
16\left(1-\frac{u^{2}}{c^{4}}\right)+4 k_{1} u=16\left(1-\frac{v^{2}}{c^{4}}\right)+4 k_{2} v .
$$

This is equivalent to

$$
\frac{4}{c^{4}}(u-v)(u+v)=k_{1} u-k_{2} v
$$


which is satisfied if and only if $k_{1}=k_{2}=16 / c^{2}$. The important feature of (3.4) is that the constant terms cancel. This only occurs with the inverse square potential, as indicated by the argument following the proof.

It remains to solve the differential equation

$$
\dot{u}^{2}=16+\frac{64}{c^{2}} u-\frac{16}{c^{4}} u^{2}
$$

for $u(t)$, after which setting $v(t)=4 c^{2}-u(t)$ will satisfy both the constraint and the differential equation for $v(t)$. By making a final change of variables $u=z+2 c^{2}$ to eliminate the linear term in the previous differential equation, we obtain

$$
\dot{z}^{2}=80-\frac{16}{c^{4}} z^{2} .
$$

This last equation has solution $z(t)=\sqrt{5} c^{2} \sin (\omega t+k)$ with $\omega=4 / c^{2}$ and $k$ an integration constant. Since varying $k$ only results in a shift of the time variable, we choose to set $k=0$. Returning to our original variables $x$ and $y$ gives the expression in the theorem.

The rhombus solution described above is defined for all time $t$ with $-\beta<t<\beta$, where $\beta=\left(c^{2} / 4\right) \sin ^{-1}(2 / \sqrt{5})$. The solution has a binary collision at $t= \pm \beta$, where the potential function is infinite. At $t=0$ the configuration becomes a square and the potential function is at a global minimum of $5 / \mathrm{c}^{2}$. As $t$ approaches $\beta, x(t) \rightarrow 2 c$ and $y(t) \rightarrow 0$ with the two bodies on the $y$-axis approaching collision. As $t$ approaches $-\beta, x(t) \rightarrow 0$ and $y(t) \rightarrow 2 c$, so the two bodies on the $x$-axis have come from collision. One can check that the value of the energy $H$ and $\dot{I}$ vanish along this orbit as well. The fact that this solution is clearly not a relative equilibrium gives

Corollary 3.4. In the equal mass 4-body problem with the inverse square potential, there exists a family of constant inertia, collision solutions that are not relative equilibria.

For completeness, we show explicitly why the rhombus counterexample requires the inverse square potential. Suppose we try to find a special rhombus solution $\mathbf{q}(t)$ as above but for a Hamiltonian system determined by the general potential function

$$
U(\mathbf{q})=\sum_{i<j} \frac{1}{r_{i j}^{\alpha}},
$$

where $\alpha>0$ is a real parameter. Since the potential function is homogeneous of degree $-\alpha$ and is also strictly positive, $\nabla U(\mathbf{q}) \neq \mathbf{0}$ and there can be no equilibrium points for the corresponding Hamiltonian system.

As before, the symmetries of our guess reduce the system to two decoupled second-order equations in the unknowns $x$ and $y$

$$
\begin{aligned}
& \ddot{x}=-2 \alpha\left(\frac{1}{x^{\alpha+1}}+\frac{x}{c^{\alpha+2}}\right), \\
& \ddot{y}=-2 \alpha\left(\frac{1}{y^{\alpha+1}}+\frac{y}{c^{\alpha+2}}\right) .
\end{aligned}
$$


Multiplying both sides of the above equations by $\dot{x}$ and $\dot{y}$, respectively, integrating once, and changing variables to $x=\sqrt{u}$ and $y=\sqrt{v}$ leads to

$$
\begin{aligned}
& \dot{u}^{2}=16\left(u^{1-\alpha / 2}-\frac{\alpha}{2 c^{\alpha+2}} u^{2}\right)+4 k_{1} u, \\
& \dot{v}^{2}=16\left(v^{1-\alpha / 2}-\frac{\alpha}{2 c^{\alpha+2}} v^{2}\right)+4 k_{2} v .
\end{aligned}
$$

Next, holding the inertia $I$ constant results in the constraint $u+v=4 c^{2}$ and the requirement that $\dot{u}^{2}=\dot{v}^{2}$. This leads to the necessary condition

$$
16\left(u^{1-\alpha / 2}-\frac{\alpha}{2 c^{\alpha+2}} u^{2}\right)+4 k_{1} u=16\left(v^{1-\alpha / 2}-\frac{\alpha}{2 c^{\alpha+2}} v^{2}\right)+4 k_{2} v .
$$

The problem with satisfying this equation is that if $\alpha \neq 2$, the terms $u^{1-\alpha / 2}$ and $v^{1-\alpha / 2}$ do not cancel out. Requiring that $u+v=4 c^{2}$, (3.5) reduces to

$$
4\left(u^{1-\alpha / 2}-v^{1-\alpha / 2}\right)-\frac{8 \alpha}{c^{\alpha}}(u-v)+k_{1} u-k_{2} v=0 .
$$

If $\alpha \neq 2$, the only possible solution to this equation subject to the constraint $u+v=4 c^{2}$ is for $u(t)=v(t) \equiv 2 c^{2}$ and $k_{1}=k_{2}$. But this results in an equilibrium solution, contradicting the fact that the system has no equilibria.

3.2. Periodic orbits. The special rhombus solution described above has zero angular momentum. It is not possible to incorporate rotations into this solution to create a periodic orbit (presumably with non-zero angular momentum) that still has constant moment of inertia. Indeed, attempts at generalizing this solution while maintaining a constant value of $I$ lead only to relative equilibrium solutions. However, non-relative equilibrium periodic solutions to the inverse square potential mechanical system are readily found using standard variational techniques dating back to the work of Poincaré [12].

Consider the configuration space of the planar $n$-body system $\mathbb{R}^{2 n}-\Sigma$, where $\Sigma$ is the set of collisions $\Sigma=\left\{\mathbf{q} \in \mathbb{R}^{2 n}: \mathbf{q}_{i}=\mathbf{q}_{j}\right.$ for some $\left.i \neq j\right\}$. Periodic solutions can be located by minimizing the action

$$
A(\gamma)=\int_{0}^{T}\left[\frac{1}{2}\|\dot{\gamma}(t)\|^{2}+U(\gamma(t))\right] d t
$$

over a given class $\Lambda$ of paths $\gamma$ in $\mathbb{R}^{2 n}-\Sigma$. For our purposes, $\Lambda$ will be some well-chosen homotopy class of absolutely continuous loops of period $T$.

There are two compactness issues which must be addressed in order to apply this variational technique successfully. One of these, coercivity, can be achieved by imposing certain constraints on the space of loops. For example, Gordon imposes homotopical constraints by tying the loops to $\Sigma$. A cycle is said to be tied to $\Sigma$ if it cannot be continuously moved off to infinity without crossing $\Sigma$ or having its length become infinite 4 . The second compactness issue is ensuring that the minimizer is free of collisions. Fortunately, the inverse square potential function is of the strong force type $(\alpha \geq 2)$ so that the action becomes infinite along any path containing a collision. This was first proved by Poincaré [12] and later elaborated upon by Gordon (see 2], p. 78). Thus, any minimizer of $A(\gamma)$ for the inverse square potential is collision-free. Since periodic solutions for the inverse square potential necessarily have constant inertia, the following theorem is an easy consequence of Gordon's work [4]. 
Theorem 3.5. Every homotopy class $\Lambda$ consisting of absolutely continuous $T$ periodic loops tied to $\Sigma$ contains a minimizer of the action $A(\gamma)$ which is collision free. Each such minimizer is a periodic solution to the inverse square potential mechanical system with constant moment of inertia.

To apply this theorem for our purposes, we need to ensure that minimizers for a given homotopy class are not relative equilibria. This is handled by the following lemma whose elementary proof was pointed out to the author by Rick Moeckel at the Equadiff conference in Hasselt, Belgium, July 22-26, 2003.

Lemma 3.6. Suppose that $\gamma_{1}$ and $\gamma_{2}$ are two loops in configuration space obtained by rigid rotation, completing $k$ rotations in time $T$. Then $\gamma_{1}$ and $\gamma_{2}$ are homotopic.

Proof. The configuration space $\mathbb{R}^{2 n}-\Sigma$ is path-connected so we can find a path $\eta(t)$ connecting $\gamma_{1}(0)$ with $\gamma_{2}(0)$ which avoids the singularity set $\Sigma$. Without loss of generality, assume that $\eta(0)=\gamma_{1}(0)$ and $\eta(1)=\gamma_{2}(0)$. Since the $\gamma_{i}$ are rigid rotations, we can simply rotate the configurations determined by the connecting path $\eta(t)$ to create a map between the two loops which avoids collisions. The continuous map

$$
F:[0, T] \times[0,1] \longmapsto \mathbb{R}^{2 n}-\Sigma, \quad F(s, t)=R\left(\frac{2 \pi k}{T} s\right) \eta(t)
$$

is a homotopy between $\gamma_{1}$ and $\gamma_{2}$, where $R(t)$ is the standard $2 \times 2$ rotation matrix (1.4). Note that we do not need to assume that the loops $\gamma_{i}$ actually correspond to relative equilibria (the position does not need to be a planar central configuration), just that the motion is a rigid rotation.

In general, to understand which homotopy classes are tied to $\Sigma$, we can associate each class with its representative from the fundamental group of $\mathbb{R}^{2 n}-\Sigma$. This group is the colored braid group on $n$ strands [11. As long as the braid type is sufficiently complicated, the corresponding homotopy class will be tied to $\Sigma$. Since relative equilibria are rigid rotations, Lemma 3.6 guarantees that any two such solutions completing $k$ rotations in time $T$ belong to the same homotopy class. Presumably, minimizing over this homotopy class will yield the relative equilibrium with the smallest action.

It follows that for each $n \geq 3$, there are an infinite number of homotopy classes $\Lambda$ tied to $\Sigma$ which do not contain relative equilibria. (This is an important distinction to make because, except for a few cases, we do not know if the number of relative equilibria equivalence classes is finite.) Each such class contains a periodic solution with constant moment of inertia, a counterexample to Saari's conjecture for the inverse square potential.

\section{ACKNOWLEDGMENTS}

The author would especially like to thank Rick Moeckel for his numerous suggestions concerning this research as well as Kuo-Chang Chen, Toshiaki Fujiwara and an anonymous referee for their insightful comments. Part of this research was supported by the American Institute of Mathematics as host of the workshop on Variational Methods in Celestial Mechanics, June 9-14, 2003. 


\section{REFERENCES}

1. A. Albouy, The symmetric central configurations of four equal masses, Hamiltonian dynamics and celestial mechanics (Seattle, WA, 1995), Contemp. Math., vol. 198, Amer. Math. Soc., Providence, RI, 1996, pp. 131-135. MR.1409157 (97g:70012)

2. A. Chenciner, Action minimizing periodic orbits in the Newtonian n-body problem, Celestial mechanics (Evanston, IL, 1999), Contemp. Math., vol. 292, Amer. Math. Soc., Providence, RI, 2002, pp. 71-90. MR1884893 (2004b:70023)

3. _ Some facts and more questions about the Eight, Topological methods, variational methods and their applications (Taiyuan, 2002), World Sci. Publishing, River Edge, NJ, 2003, pp. 77-88. MR2010643

4. W. Gordon, Conservative dynamical systems involving strong forces, Trans. Amer. Math. Soc. 204 (1975), 113-135. MR0377983 (51:14152)

5. C. Jacobi, Vorlesungen über dynamik, Reimer Publisher, Berlin, 1866.

6. C. McCord, Saari's conjecture for the planar three-body problem with equal masses, preprint, October 2002.

7. R. Moeckel, A computer-assisted proof of Saari's conjecture for the planar three-body problem, preprint, July 2003.

8. - On central configurations, Math. Z. 205 (1990), no. 4, 499-517. MR1082871 (92b:70012)

9. , Relative equilibria of the four-body problem, Erg. Th. \& Dyn. Sys. 5 (1985), no. 3, 417-435. MR.0805839 (87b:70011)

10. R. Montgomery, Action spectrum and collisions in the planar three-body problem, Celestial mechanics (Evanston, IL, 1999), Contemp. Math., vol. 292, Amer. Math. Soc., Providence, RI, 2002, pp. 173-184. MR 1884899 (2003a:70012)

11. - The $n$-body problem, the braid group, and action-minimizing periodic solutions, Nonlinearity 11 (1998), no. 2, 363-376. MR1610784 (99a:70019)

12. H. Poincaré, Sur les solutions périodiques et le principe de moindre action, Comptes Rendus 123 (1896), 915-918.

13. G. Roberts, A continuum of relative equilibria in the 5-body problem, Phys. D 127 (1999), no. 3-4, 141-145. MR1669486 (2000c:70010)

14. D. Saari, On bounded solutions of the n-body problem, Periodic Orbits, Stability and Resonances, G. Giacaglia ed., D. Riedel, Dordrecht (1970), 76-81.

15. (1980), no. 1, 9-20. MR0564603 (81a:70016)

16. S. Smale, Mathematical Problems for the Next Century, Math. Intelligencer 20 (1998), no. 2, 7-15. MR1631413 (99h:01033)

17. A. Wintner, The Analytical Foundations of Celestial Mechanics, Princeton Math. Series 5, Princeton University Press, Princeton, NJ, 1941. MR0005824 (3:215b)

Department of Mathematics and Computer Science, 1 College Street, College of the Holy Cross, Worcester, Massachusetts 01610

E-mail address: groberts@radius.holycross.edu 